\title{
Dispensação de medicamentos do componente especializado em polos no Estado do Rio de Janeiro
}

\author{
Dispensing specialized component medicines \\ in areas of the State of Rio de Janeiro
}

Elisangela da Costa Lima-Dellamora ${ }^{1}$

Rosangela Caetano ${ }^{2}$

Claudia Garcia Serpa Osorio-de-Castro ${ }^{3}$

${ }^{1}$ Faculdade de Farmácia, Universidade Federal do

Rio de Janeiro. Rua Aloísio da Silva Gomes 50, Granja dos Cavaleiros. 27930-560 Macaé RJ.

eclfar@yahoo.com.br

${ }^{2}$ Instituto de Medicina Social, Universidade do Estado do Rio de Janeiro.

${ }^{3}$ Escola Nacional de Saúde Pública, Fundação Oswaldo Cruz.

\begin{abstract}
The Specialized Pharmaceutical Services Component (CEAF) ensures the dispensing of high-cost medicines for the treatment of specif$i c$ diseases. The fact that these medicines are mandatory though access is only through legal injunctions, suggests flaws in the management of pharmaceutical services. This paper analyzed adherence to Clinical Protocols and Therapeutic Guidelines (PCDT) in the dispensing of these CEAF medicines. Qualitative research was also conducted in facilities with different characteristics in the State of Rio de Janeiro. It was noted that the lack of adequate structure in the units studied, including trained personnel, compromises the dispensing CEAF medicines in all facilities. The CEAF dispensing procedure, heavily dependent on interaction between prescribers and dispensers, is not carried out as would be expected. It is possible that inadequate performance is also linked to flaws in the planning and organization of services. The results indicate barriers in adherence to PCDT by health professionals, prejudicing health system users and possibly leading them to access medicines by legal means. It is believed that characteristics of the investigated facilities are similar to others in Brazil, and the barriers identified may be the same, compromising healthcare.
\end{abstract}

Key words Pharmaceutical assistance, Clinical protocols, Evaluation of health services
Resumo O Componente Especializado da Assistência Farmacêutica (CEAF) deve garantir dispensação de medicamentos de alto custo para o tratamento de doenças específicas. O fato desses medicamentos, previstos em listas oficiais, serem pleiteados por via judicial como estratégia de acesso à farmacoterapia sugere falhas na gestão da assistência. O presente estudo analisou a adesão aos Protocolos Clinicas e Diretrizes Terapêuticas (PCDT) no processo de dispensação de medicamentos do CEAF. Foi realizada uma pesquisa qualitativa em farmácias ambulatoriais de diferentes características no estado do Rio de Janeiro. Observou-se que a estrutura das unidades estudadas compromete a dispensação dos medicamentos do CEAF, havendo falta de recursos humanos capacitados. O fluxo de dispensação, fortemente dependente da interação entre prescritores e dispensadores, não é realizado como seria esperado. É possivel que as fragilidades no desempenho das atividades tenham origem em falhas no planejamento e na organização de serviços. Os resultados indicam barreiras na adesão dos profissionais aos PCDT, prejudicando os usuários e podendo fomentar a busca da via judicial. Estima-se que as unidades investigadas sejam semelhantes a outras no pais, e que as barreiras observadas se reproduzam, comprometendo a assistência.

Palavras-chave Assistência farmacêutica, Protocolos clínicos, Avaliação de serviços de saúde 


\section{Introdução}

Dentro do escopo da Política Nacional de Assistência Farmacêutica, o Componente Especializado da Assistência Farmacêutica (CEAF) é uma estratégia de acesso aos medicamentos voltados à atenção de média e alta complexidade, no âmbito do Sistema Único de Saúde, que busca garantir a integralidade do tratamento medicamentoso, em nível ambulatorial ${ }^{1,2}$. Anteriormente denominados Componente de Medicamentos de Dispensação Excepcional, os fármacos relacionados para este tipo de atenção foram redefinidos como CEAF com a publicação da Portaria GM/MS n 2981 de 30 de novembro de $2009^{3}$.

O CEAF concentra incorporação de medicamentos novos e por vezes caros, o que pressupõe sua adequada utilização na prática assistencial para obtenção dos resultados pretendidos. Nesse sentido, Protocolos Clínicos e Diretrizes Terapêuticas (PCDT) foram elaborados e publicados pelo Ministério da Saúde. O estabelecimento de diretrizes técnico-administrativas e gerenciais para situações clínicas prioritárias (por sua prevalência, complexidade ou impacto financeiro) para a saúde pública visou difundir em larga escala a informação técnica e de qualidade baseada na melhor evidência científica existente ${ }^{1}$.

Nos PCDT constam os critérios de diagnóstico, o algoritmo de tratamento das doenças, os mecanismos para o monitoramento clínico e a supervisão de possíveis efeitos adversos a medicamentos. No que tange aos profissionais responsáveis pela dispensação, esse documento recomenda e fornece elementos para a implementação de serviços voltados à prática de um modelo fundamentado na Atenção Farmacêutica. Propõe a interação direta do farmacêutico com o usuário visando uma farmacoterapia racional e a obtenção de resultados definidos e mensuráveis, com melhora na qualidade de vida ${ }^{1,4}$.

Neste aspecto, a falta de adesão aos PCDT contribuiria para a descontinuidade das diretrizes formuladas, interferindo na qualidade da assistência prestada ${ }^{5}$. De fato, os achados da literatura quanto à tentativa de acesso aos medicamentos do CEAF por via judicial nos estados do Rio de Janeiro ${ }^{6,7}$ e Santa Catarina ${ }^{8,9}$, no município de São Paulo ${ }^{10,11}$ e no Distrito Federal ${ }^{12}$ sugerem falhas na gestão deste Componente, apontando para a possibilidade de problemas estruturais e de adesão às diretrizes.

A execução do CEAF ocorre em farmácias espalhadas por todo país, denominadas polos, que tramitam a solicitação dos medicamentos (cadastro de pacientes), a avaliação, a renovação e a autorização dos pedidos e a dispensação dos medicamentos $^{1}$. O funcionamento dos polos é particularmente importante. A estruturação e a gestão desses locais, incluindo os requisitos mínimos para dispensação de medicamentos esperados e previstos na legislação brasileira ${ }^{13}$, devem estar implantados, podendo pautar a efetividade do CEAF com implicações sobre a organização da assistência farmacêutica ${ }^{14}$.

Considerando a importância do CEAF no acesso aos tratamentos com medicamentos de custo elevado, o presente estudo objetivou examinar a dispensação em polos no estado do Rio de Janeiro, durante os anos 2008 e 2009, verificando a adesão dos profissionais responsáveis aos PCDT. Espera-se que o exame dos locais estudados possibilite uma aproximação com a dispensação de CEAF em outros polos do país.

\section{Metodologia}

Foi realizado um estudo exploratório de abordagem metodológica qualitativa, em locais (polos) de dispensação de medicamentos do componente especializado localizados no estado do Rio de Janeiro.

À época do estudo, existiam trinta e seis polos de dispensação no estado. Onze encontravam-se nas cidades do Rio de Janeiro e Niterói: farmácia central do estado, hospitais de grande porte e institutos especializados. Outros vinte e cinco se distribuíam nos demais 90 municípios do estado, em farmácias municipais, postos de saúde ou hospitais de pequeno porte. A escolha do campo tomou por base uma amostra intencional que contemplou diferentes perfis e demandas dos polos, bem como maior ou menor interação com a SESDEC-RJ, conforme a participação dos farmacêuticos em treinamentos e/ou por contatos telefônicos frequentes, segundo a própria Secretaria. Desta forma, foram selecionadas, para visita e demais etapas da investigação, as farmácias ambulatoriais de dois hospitais gerais (sendo um deles universitário), de um posto de saúde de um município da Baixada Fluminense, e do polo central estadual localizado na capital do estado.

Para a investigação do processo de dispensação de medicamentos do componente especializado previsto nos PCDT, foram empregadas três estratégias complementares: (i) coleta de dados por meio de análise documental, incluindo os documentos previstos nos PCDT e outros especí- 
ficos das Unidades relativos a dispensação de medicamentos do CEAF; (ii) observação direta do processo de dispensação nas Unidades, e (iii) aplicação de um questionário semiestruturado aos farmacêuticos lotados nos locais selecionados.

Foram desenvolvidos dois instrumentos de coleta de dados empíricos: um orientado a observação do campo e outro para as entrevistas com atores chave. A formulação destes dois baseou-se nas condutas indicadas nos PCDT que especificam o processo de dispensação de medicamentos, quais sejam: fluxograma de dispensação que inclui solicitação de medicamento; análise pelo dispensador; dispensação do medicamento e o acompanhamento farmacoterapêutico, registrado em fichas farmacoterapêuticas. O modelo para cada medicamento é disponibilizado em protocolos específicos que compõem os PCDT $^{1}$.

Considerando as implicações do contexto para a atividade, os instrumentos também contemplaram aspectos relacionados às boas práticas de dispensação ${ }^{14,15}$, como as condições estruturais dos polos - local de atendimento, recursos humanos, gerenciamento do fluxo e da demanda do polo, definição e identificação da documentação; habilitação e qualificação do corpo funcional - e a interação entre os profissionais ${ }^{16}$.

Foi realizado o pré-teste dos instrumentos em um instituto estadual especializado, polo de dispensação de medicamentos do CEAF, tendo sido promovidos pequenos ajustes na forma e especificação das questões originais. Este novo instrumento ajustado foi aplicado em um piloto conduzido em um hospital estadual de grande porte, também polo de dispensação, com a finalidade de mimetizar todas as etapas do trabalho empírico.

A coleta de dados somente foi iniciada após a leitura, aceite e assinatura de Termo de Consentimento Livre Esclarecido pelos entrevistados. A pesquisa obteve aprovação do Comitê de Ética em Pesquisa do Hospital Universitário Clementino Fraga Filho (RJ).

As visitas foram agendadas por telefone com o farmacêutico responsável pela dispensação nos Polos selecionados e todas realizadas pelo mesmo pesquisador

O número de entrevistas foi definido através do processo de inclusão progressiva (sem demarcação prévia), limitada por critério de saturação. Cada entrevista foi transcrita logo após a sua realização, o que permitiu maior clareza dos conteúdos trazidos pelas respostas dos farmacêuticos arrolados no trabalho. Quando se identificou que as novas entrevistas começaram a apre- sentar regularidade nos conteúdos, deixando de agregar novas informações ${ }^{17}$, considerou-se que já era possível a sua interrupção.

Os dados descritivos obtidos foram tabulados e analisados de modo a possibilitar a construção do perfil assistencial dos polos envolvidos. Os documentos foram organizados por tipo e assunto, de modo a permitir uma melhor compreensão e corroboração dos relatos dos entrevistados e das observações da dispensação. As informações obtidas por observação direta foram integradas ao conjunto de elementos advindos das estratégias acima mencionadas.

As entrevistas forneceram o discurso dos atores-chave envolvidos com o CEAF. Foi utilizada a técnica de análise de conteúdo proposta por Bardin $^{18}$. A análise documental forneceu um mapa inicial de pré-categorias, adequadas àquelas presentes nos PCDT e que ajudou a conduzir a leitura flutuante das falas presentes nas entrevistas. A partir da leitura exaustiva dessas falas, núcleos de sentido foram identificados e desmembrados, de modo a possibilitar o surgimento das seguintes categorias temáticas finais: (a) condições estruturais dos polos, (b) adesão ao processo de dispensação previsto nos PDCT, e (c) interação entre os profissionais envolvidos.

\section{Resultados e discussão}

\section{Condições estruturais dos polos de dispensação}

A estrutura e a organização de todos os polos visitados afetavam a dispensação dos medicamentos do CEAF, por não atenderem aos requisitos mínimos de boas práticas de dispensação. Três, das quatro farmácias visitadas, mesmo apresentando área para dispensação em desacordo ao recomendado ${ }^{14}$, se responsabilizavam adicionalmente pela dispensação de medicamentos de outros programas.

Sabe-se que fatores estruturais que comprometem a visualização e a compreensão da prescrição dificultam seu aviamento e impedem a orientação, além de oportunizar erros de medicação ${ }^{14}$. Em todos os polos, os espaços destinados ao arquivo de documentos, à guarda de medicamentos e ao atendimento eram insuficientes, com pouca iluminação e muito barulho. Em dois deles, a dispensação realizava-se por uma “janela”, o que dificultava a entrega da documentação necessária para solicitação dos medicamentos e a orientação dos usuários quanto ao uso. Do 
momento que a interação com o paciente é medida necessária ao dispensador, a distância física e a falta de privacidade inviabilizam, de fato, a efetividade do cuidado farmacêutico ${ }^{4}$.

Todas as farmácias visitadas possuíam, pelo menos, um computador. Porém, o número e a condição de funcionamento desses equipamentos se mostraram insuficientes e inadequados, tornando a dispensação demasiadamente lenta. A Tabela 1 mostra detalhes, entre outros, do atendimento aos usuários do CEAF, evidenciando que o tempo de atendimento em alguns polos é um problema importante. A falta de outros equipamentos, como impressoras, e de material de escritório, comprometia também a realização das atividades administrativas relacionadas ao CEAF, entre as quais a prestação de contas à Secretaria Estadual de Saúde e Defesa Civil e a solicitação dos medicamentos.

Em duas unidades, houve relato de falta de meios de transporte para o encaminhamento da documentação ao órgão estadual, o que contribuía para irregularidade de fornecimento e, até mesmo, falta de medicamentos nos polos.

Centrar o foco no paciente e não no produto é condição de melhoria de qualidade dos processos em saúde, ligados aos desfechos clínicos pretendidos ${ }^{1,4}$. Não se identificou nenhum documento de procedimento operacional padrão (POP) que correspondesse às atividades relacionadas à dispensação, o que significa que não é possível a definição de padrões mínimos a serem seguidos pela equipe, nem sua monitoração, uma vez estabelecidos. Isto contrasta com toda a proposta de atenção subjacente aos PCDT. Foi relatada também a inexistência de treinamento formal para os profissionais em todos os polos visitados. Estas carências de qualificação podem gerar informações e hábitos inadequados às atividades de assistência farmacêutica ${ }^{16}$, mormente aquelas diretamente ligadas aos usuários.
No que tange à supervisão, todos os polos visitados afirmavam possuir um farmacêutico responsável pela dispensação dos medicamentos. Entretanto, este profissional não se encontrava presente regularmente em dois destes polos, demonstrando o não cumprimento dos aspectos legais e das diretrizes do Componente $e^{1,10}$. A carga horária semanal dos farmacêuticos e dos profissionais de nível médio observada estava inferior à necessária para atender de forma adequada ao número de pacientes cadastrados no CEAF e nos demais programas, quando existentes, considerando os parâmetros mínimos para recursos humanos estabelecidos pela Sociedade Brasileira de Farmácia Hospitalar ${ }^{19}$. As informações sobre os recursos humanos disponíveis nas Unidades estão também dispostas na Tabela 1.

Foram entrevistados quatro profissionais, todos farmacêuticos responsáveis pelos polos, com idade entre 25 a 38 anos. O tempo de formação variou entre dois a nove anos. Um farmacêutico havia concluído um curso de pós-graduação lato sensu em Farmácia Hospitalar. Os entrevistados relataram possuir entre um e cinco anos de atividade nas unidades estudadas; entretanto, o período de responsabilidade pelos polos era bem menor: três a dezoito meses.

O número total de farmacêuticos lotados no polo não era proporcional sequer ao montante de pacientes atendidos, quiçá às atividades demandadas pelos PCDT. Esta variação de parâmetros de atendimento nos polos é também causa provável de discrepâncias na atenção integral aos pacientes, com possíveis consequências sobre os desfechos clínicos esperados. Outra possibilidade é o descrédito do serviço, levando o usuário a buscar outras vias de acesso ao medicamento, entre elas a administrativa e a judicial, que por sua vez oneram a gestão, em recursos financeiros e humanos.

O CEAF parece não estar contribuindo para a mudança na tendência de incremento de ações

Tabela 1. Caracterização da demanda e recursos humanos das farmácias ambulatoriais visitadas entre 2008 e 2009.

\begin{tabular}{ccccccc}
\hline Farmácia & $\begin{array}{c}\text { No de } \\
\text { pacientes } \\
\text { cadastrados }\end{array}$ & $\begin{array}{c}\text { Tempo de espera } \\
\text { para atendimento } \\
\text { (em minutos) }\end{array}$ & $\begin{array}{c}\text { No de }_{\text {farmacêuticos/ }} \\
\text { dia }\end{array}$ & $\begin{array}{c}\text { No de }_{\text {profissionais de }} \\
\text { nível médio/dia }\end{array}$ & $\begin{array}{c}\text { Área para } \\
\text { dispensação } \\
\left(\text { em m }^{2}\right)\end{array}$ & $\begin{array}{c}\text { Dispensação exclusiva } \\
\text { de medicamentos do } \\
\text { CEAF }\end{array}$ \\
\hline A & 70 & Até 10 & 1 & 1 & 8,8 & NÃO \\
B & 600 & 10 a 80 & $1^{*}$ & 2 & 11 & NÃO \\
C & 3.000 & 30 a 90 & $1^{*}$ & 2 & 18,5 & NÃO \\
D & 15.000 & 30 a 300 & $4^{* *}$ & 4 & 150 & SIM \\
\hline
\end{tabular}

Observações: ${ }^{*}$ ausência de farmacêutico em um dia da semana ou turno. ${ }^{* *}$ possuía um administrador responsável pela programação. 
judiciais no país. Blatt e Farias ${ }^{8}$ observaram deficiências importantes no estado de Santa Catarina quanto à infraestrutura e pessoal, condições de armazenamento e de atendimento durante a dispensação do CEAF. No Rio de Janeiro, em especial, parte considerável dos medicamentos demandados judicialmente são do $\mathrm{CEAF}^{6}$.

Em 2004, o Conselho Nacional de Secretários de Saúde (CONASS) reiterou que os serviços necessários à operacionalização do CEAF deveriam ser estruturados e organizados de forma a atender aos objetivos propostos ${ }^{20}$.

Deve-se ressaltar que os PDCT, na medida em que estão centrados nas atividades necessárias a adequada dispensação e não mencionam as condições estruturais mínimas para que esta se dê de acordo com as boas práticas relatadas na literatura, podem induzir a falhas de atenção. A gestão do CEAF precisa levar em conta não apenas o disposto nos PDCT, mas todas as necessidade em termos de infraestrutura, recursos humanos e serviços de apoio que permitam a sua implementação efetiva.

De acordo com os farmacêuticos entrevistados, as necessidades estruturais e de recursos humanos, incluindo desde aquelas para as atividades mais simples até os procedimentos mais complexos, ainda não haviam sido alcançadas nos polos visitados. A esse respeito, os profissionais foram unanimemente críticos quanto a estas deficiências, frente ao altíssimo custo e impacto clínico associado aos medicamentos em pauta, a ponto de um dos entrevistados afirmar:

Eles só se preocupam em comprar o medicamento, mas não onde ele será colocado ou no que a gente precisa nos serviços (entrevistado 3 ).

\section{Adesão ao processo de dispensação preconizado nos PCDT}

Sobre as recomendações dos PCDT para o momento da dispensação - confirmação dos critérios de inclusão e exclusão previstos, verificação dos exames obrigatórios para abertura do processo, análise do correto preenchimento do Laudo para Solicitação e Autorização de Medicamentos (LME) do CEAF e da dose prescrita - os profissionais alegaram que o recebimento da documentação na farmácia estava focado no correto preenchimento da LME, sem uma avaliação aprofundada relativa aos exames, aos critérios de inclusão e exclusão e à dose prescrita. Nenhuma das farmácias visitadas apresentou a utilização da carta-modelo, das fichas de registro ou farmacoterapêutica, ou do guia de orientação ao paciente, sugeridos pelos $\mathrm{PCDT}^{1}$. Questionados, os farmacêuticos responderam:

A gente só confere a LME mesmo. Quem tem que se preocupar com os exames e os critérios de inclusão é o médico. A responsabilidade é deles (entrevistado 3).

Nós não temos farmacêuticos suficientes para fazer o acompanhamento farmacoterapêutico (entrevistado 2).

Em todos os polos, os PCDT estavam acessíveis para consulta. As propostas de acompanhamento farmacoterapêutico que integram o PCDT ensejam o farmacêutico a aderir a tarefas administrativas e clínicas. Não foi observado, contudo, um fluxo de atendimento correspondente ao previsto e, sim, o descumprimento destas atividades. Diante dos resultados, é inevitável que todos os farmacêuticos entrevistados se considerassem despreparados para realizar Atenção Farmacêutica, campo de prática que reconhecidamente congrega muitos requisitos técnicos, de habilidades e atitudes ${ }^{4}$. Um dos motivos observados seria a carência dos polos em requisitos de estrutura física e de capacitação, já discutidos. No entanto, embora o acesso prévio ao prontuário médico pelo farmacêutico fosse possível em dois polos, além de recomendado pelos PCDT, os profissionais dessas unidades demonstravam dificuldade ou pouco interesse em fazê-lo.

Em sua primeira edição, publicada em 2002, no que tange aos profissionais dispensadores e à prática do acompanhamento farmacoterapêutico, os PCDT sugeriram a necessidade de "mudança de enfoque filosófico, organizacional e funcional da farmácia, elevando o seu nível de responsabilidade e do profissional farmacêutico"2. Apesar de sinalizadas, as mudanças aludidas não parecem ter sido cumpridas, pelo menos nos polos visitados.

A falta de participação conjunta no processo de formulação de políticas, protocolos e diretrizes provoca estranhamento entre os atores responsáveis por sua elaboração e os profissionais que promovem sua implementação ${ }^{5}$. No caso dos PCDT, os prescritores e os dispensadores, por vezes, são responsabilizados pela falta de êxito de uma política com a qual podem não ter contribuído ${ }^{5,21,22}$. Neste contexto, mesmo que os PCDT tenham objetivado criar mecanismos para garantia da prescrição segura e eficaz, contemplando um acompanhamento realizado pelo farmacêutico ${ }^{1}$, muitas vezes ainda não se observa a real participação deste profissional no processo.

A motivação parece estar mesmo bastante imbricada no desempenho observado nas uni- 
dades. Os farmacêuticos mencionaram o desgaste no desenvolvimento das atividades do CEAF, utilizando termos como raiva, impaciência, descaso e cansaço nos relatos sobre o processo de dispensação.

Eu sou contratada para 20 horas semanais e fico aqui todo dia de manhã. Na semana de dispensação de interferon, eu só consigo ir embora depois das seis da tarde. Não dá tempo de fazer mais nada. E, mesmo assim, os pacientes brigam numa fila enorme lá fora [...] o pior é que eles só sentem raiva da farmácia (entrevistado 2).

Aqui, você só vê descaso da administração. Eles jogam o programa em cima da gente sem a menor estrutura [...] você acha que alguém tem a paciência de esperar um atendimento diferenciado pelo farmacêutico com esta falta de profissionais? (entrevistado 3).

Aqui tem essa fila todo dia. Às vezes, está pior. Vai melhorar porque uma farmácia nova está sendo construída. Mas hoje, é muito cansativo. Para nós e para o paciente (entrevistado 4).

Além disso, estes profissionais percebem sua participação no CEAF como eminentemente burocrática. A multiplicidade de tarefas e a desestruturação física do serviço não explicam o observado em uma unidade, onde o farmacêutico acrescentou que

[...] o tempo que a gente iria perder fazendo a atenção farmacêutica atrasaria muito o paciente (entrevistado 3).

É interessante verificar que a percepção referida pode estar vinculada ao pequeno tempo de formado dos entrevistados, bem como ao curto período de participação em atividades clínicas e, mormente, na responsabilidade pelos polos. $\mathrm{O}$ trabalho considerado "burocrático" é, muitas vezes, um sustentáculo para o acompanhamento dos pacientes e para a atividade clínica. Esta percepção pode, portanto, ser consequência da relativa inexperiência desses profissionais.

Em duas unidades, o CEAF foi referido como um "peso e mais uma atividade burocrática" para os profissionais em meio a tantas atribuições, questionando-se a sua importância.

[...] a gente já fazia a dispensação de DST/AIDS e dos oncológicos aqui. Com os medicamentos excepcionais, ficou impossível! Não tem computadores nem funcionários para fazer os relatórios mensais. É muito pesado. Eu levo os documentos para terminar em casa ou no meu outro trabalho (entrevistado 3).

O acompanhamento farmacoterapêutico ainda é incipiente no Brasil ${ }^{23}$, mesmo que os PCDT tenham apresentado um modelo de prá- tica para o Componente Especializado. As concepções do farmacêutico quanto à assistência farmacêutica estão focadas nas atividades de gestão ${ }^{24,25}$ desarticuladas da clínica. Ainda que a dependência entre essas atividades estruturantes e o papel que a Atenção Farmacêutica deve desempenhar sejam referidos na literatura ${ }^{23,24}$, os resultados do estudo mostram que, nos serviços visitados, não há suficiente amadurecimento para adesão da proposta do PCDT.

\section{Interação entre profissionais}

A troca de informações sobre os medicamentos e os pacientes não deveria ser incomum entre médicos e farmacêuticos, diante da importância do desfecho para o usuário ${ }^{16,25}$. Esta interação, entretanto, tem se mostrado tumultuada e os polos do CEAF não diferiram neste aspecto.

A primeira dificuldade diz respeito às formas de relacionamento adotadas e à falta de proximidade entre serviços médicos e farmacêuticos. Em três polos, a ocorrência de algum problema com a documentação do paciente resultava em retorno de ordem verbal ao médico; em outro, a orientação era dada de forma escrita, devido à localização do ambulatório, em um prédio diferente daquele onde ocorria a dispensação. A carta-modelo sugerida pelo PCDT para esta comunicação não foi mencionada em nenhuma das quatro unidades examinadas.

No contexto da saúde, profissionais envolvidos no cuidado citam as condições de trabalho, a falta de capacitação, a baixa remuneração e o relacionamento conflituoso como limites, muitas vezes, intransponíveis ${ }^{26}$. Eles não se enxergam como sujeitos de um processo de mudança para a melhoria da assistência prestada. Como resultado, são criados, nesses ambientes, processos paralelos ou alternativos, de cunho individual, para a resolução de problemas ${ }^{26}$, que podem se contrapor aos processos de trabalho que melhor contribuiriam para a proteção à saúde.

O individualismo no enfrentamento dos problemas e as dificuldades do trabalho em equipe são exemplificados pelos farmacêuticos entrevistados no contato com os médicos, em relação a dúvidas na prescrição e ao preenchimento inadequado ou incompleto dos documentos necessários para a dispensação dos medicamentos do CEAF. Em duas unidades, foi relatado que os prescritores se negaram a fazer tais correções, quando solicitados. Foi mencionado que um médico informou ao paciente que estava mudando a prescrição para um medicamento que não 
necessitava de LME "porque a farmácia criava muitos problemas”. Em outro caso, o médico teria se negado a alterar a LME, que só foi refeita ao cair em exigência, após envio à SESDEC, ocasionando um atraso de 35 dias na dispensação.

A percepção de que as dificuldades e os entraves na dispensação prejudicam os pacientes e geram retrabalho pode ser expressa na atitude de alguns prescritores, ignorando as consequências sobre a gestão e o coletivo. A formação de estoques caseiros pelos pacientes, pretensamente sugeridos pelos médicos assistentes, foi descrita como uma forma de "driblar" eventuais faltas de medicamentos.

[...] o que acontece muito aqui é o médico pedir o dobro da dose e pedir para o paciente guardar (entrevistado 1).

A execução do CEAF parece conjugar a limitação de conhecimentos dos profissionais e o direcionamento do foco ao medicamento e não ao paciente. Um farmacêutico afirmou que, apesar do protocolo para tratamento de determinada doença preconizar o uso associado de dois fármacos, metade dos pacientes apresentava prescrições só contendo um deles.

A fragilidade na formação clínica do farmacêutico no Brasil ${ }^{23,27}$ não ajuda a estabelecer uma relação de igualdade e de parceria com o prescritor, o que teria oportunizado uma intervenção efetiva em favor do paciente. Quando interpelado sobre sua atitude em relação à prescrição inadequada, o farmacêutico respondeu que acatou a justificativa do prescritor, de que

[...] um trabalho científico indicava o uso de apenas um fármaco e que o PCDT não deveria ser considerado (entrevistado 1).

Os relatos também trouxeram à pauta a falta de entendimento dos farmacêuticos em relação a suas responsabilidades frente aos demais profissionais e ao papel da gestão central. Respondendo sobre a discordância entre a dose prescrita e a preconizada, um farmacêutico alegou que

o médico assina um termo de responsabilidade [...] não sou eu que vou fazer o trabalho da SESDEC (entrevistado 3).

As ocorrências durante a interação entre os diversos atores indicam a importância de compreensão do caráter humano e social que influencia os processos organizacionais. A expressão de equívocos e incertezas advindas de novas formas de organização do trabalho no serviço de saúde, como o proposto nos PCDT, sugere a necessidade de enfoque no papel dos sujeitos neste processo $^{28}$. Entende-se como um investimento imprescindível a promoção de estratégias que busquem a prática interdisciplinar e aproxime as formas de organização do trabalho, favorecendo o vínculo e o acolhimento dos profissionais ${ }^{29}$.

A respeito dos problemas nas relações, Cecílio $^{30}$ refere à tensão entre controle versus autonomia no processo de gestão, quanto à temática do poder, particularmente em hospitais. Estes conflitos estão no âmago das dificuldades relatadas nos polos, e merecem atenção.

A estrutura de autoridade, por exemplo, é também descrita como dúbia nos hospitais, por trazer linhas de comando com lógicas e interesses diferentes. Serviços administrativos e de suporte seriam mais voltados a interesses econômicos e uma lógica inversa referir-se-ia ao cuidado profissional prestado ${ }^{31}$. Os serviços farmacêuticos nos PCDT traduzem, em parte, esta ambiguidade. Apesar de terem como responsabilidade um atendimento clínico no âmbito da farmacoterapia, os farmacêuticos entendem sua atividade como burocrática, por suas diversas funções administrativas ${ }^{23}$, percepção esta que é compartilhada com os prescritores.

Diante de uma proposta de acompanhamento farmacoterapêutico que se realiza junto ao paciente e ao prescritor, a adoção de um novo modo de se fazer assistência não se impõe naturalmente ${ }^{30}$. Cabana et al. ${ }^{32}$ investigaram 293 potenciais barreiras para a adesão a protocolos. Segundo estes autores, existem obstáculos relacionados ao conhecimento (falta de familiaridade e de atenção aos protocolos), à atitude (expectativas quanto ao resultado, entendimento, motivação, confiança insuficientes) e ao comportamento (barreiras externas relacionadas ao paciente, aos protocolos e ao ambiente, tais como falta de tempo e recursos, e constrangimentos organizacionais). Similares obstáculos emergiram no presente estudo quanto à adesão aos PCDT pelos farmacêuticos.

O grau de adesão aos protocolos é influenciado por um grande número de fatores, relacionados à própria diretriz, aos esforços realizados pelas organizações profissionais, à gestão do cuidado e às características do profissional e dos pacientes $^{33}$. Forsner et al. ${ }^{34}$ mensuraram que o cumprimento de diretrizes clínicas por psiquiatras foi maior depois de um processo ativo de implementação que incluiu seminários, equipe local de suporte e visitas acadêmicas. Grol e Grimshaw $^{35}$ sugerem discussões permanentes entre os profissionais quanto aos resultados da mudança de prática, consensos e comentários sobre o desempenho por pares, em um estudo sobre a introdução de diretrizes clínicas para médicos. 
Para estes autores, são necessárias abordagens abrangentes em diferentes níveis - indivíduo, equipe e instituição - de modo a ampliar o grau de adesão dos profissionais.

A literatura é pobre na oferta de estudos que discutam a aderência a protocolos e diretrizes por farmacêuticos. Sabe-se, no entanto, que estratégias de fomento ao uso racional de medicamentos se apoiam firmemente na atuação do farmacêutico junto ao paciente e à equipe de saú$\mathrm{de}^{36}$. Tendo em vista a importância desse profissional na orientação aos pacientes submetidos a tratamentos protocolizados ou embasados em diretrizes, justifica-se este estudo, que pretende agregar elementos determinantes para a modificação dessa situação.

\section{Considerações finais}

Apesar de referir-se apenas às unidades examinadas, os resultados encontrados podem permitir algumas considerações quanto à forma geral de comportamento dos profissionais farmacêuticos em polos de dispensação, na medida em que as concepções, as explicações e os sentidos atribuídos pelos dispensadores tiveram uma regularidade de apresentação nas entrevistas.

Acredita-se que a adesão dos profissionais dispensadores aos PCDT e, em especial, à realização do acompanhamento farmacoterapêutico, está fortemente condicionada pelas condições estruturais de dispensação destes medicamentos, pela formação e cultura dos farmacêuticos, pela relação com outros profissionais e pela política interna dos serviços. Além de estar dificultada por barreiras relativas ao conhecimento, às atitudes e ao comportamento dos profissionais, observa-se ainda que a responsabilidade de dispensação de medicamentos do CEAF, com todas as suas particularidades, é dada aos polos, sem a contrapartida relativa à organização e ao planejamento dos serviços.

Reconhece-se que a complexidade do preenchimento destes vazios estruturais nos serviços vai além do fomento e da programação. Os elementos apresentados são agravados ainda pela forma como se relacionam os atores no ambiente organizacional. Não surpreende que os usuários do CEAF encontrem dificuldades no acesso à farmacoterapia, diante do diagnóstico das condições de assistência farmacêutica que culminam na não adesão aos PCDT. Estima-se que as dificuldades de gestão do CEAF possam estar influenciando o incremento de vias alternativas de acesso a medicamentos do componente, entre elas a via judicial.

As reflexões apresentadas neste trabalho, sobre (i) a estrutura necessária às boas práticas de dispensação destes medicamentos, (ii) a obrigatoriedade de execução de etapas documentais e funcionais, sem que se possua governabilidade, autoridade, disponibilidade ou conhecimento para fazê-lo, e (iii) as tensões nas relações entre os atores que promovem a assistência, são prementes para a discussão da proposta de mudança do enfoque filosófico, organizacional e funcional da unidade de dispensação, sugerido pelos PCDT. Uma transformação neste sentido poderia contribuir para minimizar a demanda judicial para o fornecimento de medicamentos já selecionados em listas públicas, e realmente modificar as características de uso dos medicamentos do CEAF. 


\section{Colaboradores}

EC Lima-Dellamora foi responsável pela coleta, análise e discussão dos dados e redação do artigo. CGS Osório-de-Castro pela orientação de todas as etapas e redação do artigo; R Caetano colaborou na discussão dos dados e revisão crítica do texto.

\section{Agradecimentos}

Às professoras Vera Lucia Luiza e Elizabeth Artmann pelas valiosas colaborações no planejamento do estudo e na revisão crítica do manuscrito, respectivamente. À Secretaria Estadual de Saúde e Defesa Civil do estado do Rio de Janeiro pelo fomento à pesquisa.

\section{Referências}

1. Brasil. Ministério da Saúde (MS). Protocolos Clínicos e Diretrizes Terapêuticas. Brasília: MS; 2010.

2. Picon PD, Beltrame A. Protocolos Clínicos e Diretrizes Terapêuticas. 2002. [site na Internet]. [acessado em 2011 abr 29]. Disponível em: http://dtr2001. saude.gov.br/sas/dsra/protocolos/index.htm.

3. Brasil. Ministério da Saúde (MS). Portaria GM/MS $n^{\circ} 2981$ de 30 de novembro de 2009. Aprova o Componente Especializado da Assistência Farmacêutica. Diario Oficial da União 2009; 30 nov.

4. Ivama AM, Noblat L, Castro MS, Oliveira NVBV, Jaramillo NM, Rech N. Consenso Brasileiro de Atenção Farmacêutica: Proposta. Brasília: Organização Pan-Americana da Saúde; 2002.

5. Sampaio J, Araújo-Junior JL. Análise das políticas públicas: uma proposta metodológica para o estudo no campo da prevenção em Aids. Rev. Bras. Saúde Matern. Infant 2006; 6(3):335-346.

6. Messender AM, Osório-de-Castro CGS, Luiza VL. Mandados judiciais como ferramenta para garantia do acesso a medicamentos no setor público: a experiência do estado do Rio de Janeiro, Brasil. Cad Saude Publica 2005; 21(2):525-534.

7. Santana JMB. Essencialidade e Assistência Farmacêutica: um estudo exploratório das demandas judiciais individuais para acesso a medicamentos no estado do Rio de Janeiro. [dissertação]. Rio de Janeiro (RJ): Fiocruz; 2009.

8. Blatt CR, Farias MR. Diagnóstico do Programa de Medicamentos Excepcionais do Estado de Santa Catarina - Brasil. Lat. Am. J. Pharm. 2007; 26(5):776783.

9. Pereira JR, Santos RI, Nascimento-Junior JM, Schenkel EP. Análise das demandas judiciais para o fornecimento de medicamentos pela Secretaria de Estado da Saúde de Santa Catarina nos anos de 2003 e 2004. Cien Saude Colet 2010; 15(Supl. 3): 3551-3560.

10. Chieffi AL, Barata RB. Judicialização da política pública de assistência farmacêutica e eqüidade. Cad Saude Publica 2009; 25(8):1839-1849.

11. Vieira FS, Zucchi P. Distorções causadas pelas ações judiciais à política de medicamentos no Brasil. Rev Saude Publica 2007; 41(2):214-222.

12. Romero LC. Judicialização das políticas de assistência farmacêutica: o caso do distrito federal. Brasília: Consultoria Legislativa do Senado Federal; 2008. [página na Internet]. [acessado 2010 out 30]. Disponível em: http://www.senado.gov.br/senado/coleg/textos_discussão/

13. Brasil. Resolução ANVISA no 328 de 22 de julho de 1999. Dispõe sobre requisitos exigidos para a dispensação de produtos de interesse à saúde em farmácias e drogarias. Diário Oficial da União 1999; 26 jul.

14. Marin N, Luiza VL, Osório-de-Castro CGS, Machado-dos-Santos S, organizadores. Assistência Farmacêutica para gerentes municipais. Brasília: Organização Pan-Americana de Saúde; 2003.

15. Angonesi D. Dispensação farmacêutica: uma análise de diferentes conceitos e modelos. Cien Saude Colet 2008; 13(Supl. 1):629-640. 
16. Pepe VLE, Osório-de-Castro CGS. A interação entre prescritores, dispensadores e pacientes: informação compartilhada como possível benefício terapêutico. Cad Saude Publica 2000; 16(3):815-822.

17. Minayo MCS, Trabalho de campo: contexto de observação, interação e descoberta. In: Deslandes SF, Gomes R, Minayo MCS, organizadores. Pesquisa social: teoria, método e criatividade. Petrópolis: Vozes; 2008. p. 61-77.

18. Bardin L. Análise de Conteúdo. Lisboa: Edições 70; 2007.

19. Sociedade Brasileira de Farmácia Hospitalar (SBRAFH). Padrões Mínimos para Farmácia Hospitalar. Goiânia: SBRAFH; 2007.

20. Brasil. Conselho Nacional de Secretaria de Saúde (CONASS). Para entender a gestão do Programa de Medicamentos dispensação em caráter excepcionais. Brasília: CONASS Documenta; 2004.

21. Carvalho LJMA. Sobre a Política de Dispensação de Medicamentos no Brasil. Revista Direito e Justiça. 2008; 11:161-169.

22. Krauss-Silva L. Avaliação tecnológica e análise custo-efetividade em saúde: a incorporação de tecnologias e a produção de diretrizes clínicas para o SUS. Cien Saude Colet 2003; 8(2):501-520.

23. Araújo ALA, Freitas O. Concepções do profissional farmacêutico sobre a assistência farmacêutica na unidade básica de saúde: dificuldades e elementos para a mudança. Braz Journal Pharm Sciences 2006; 42(1):137-146.

24. Araújo ALA, Pereira LRL, Ueta JM, Freitas O. Perfil da Assistência Farmacêutica na atenção primária do Sistema Único de Saúde. Cien Saude Colet 2008; 13 (Supl. 1):611-617.

25. Penaforte TR, Forster AC, Simões MJS. Evaluations of the performance of pharmacists in terms of providing health assistance at a university hospital. Clinics 2007; 62(5):567-572.

26. Mendes AWB, Caldas Junior AL. Prática Profissional e Ética no Contexto das Políticas de Saúde. Rev Latino-am. Enfermagem. 2001; 9(3):20-26.

27. Castro MS, Correr CJ. Pharmaceutical care in community pharmacies: practice and research in Brazil. The Annals of Pharmacoterapy 2007; 41(9):14861493.
28. Azevedo CS. A dimensão imaginária e intersubjetiva das organizações de saúde: implicações para o trabalho gerencial e para a mudança organizacional. Cien Saude Colet 2010; 15(Supl. 1):977-986.

29. Matos E, Pires DEP, Campos GWS. Relações de trabalho em equipes interdisciplinares: contribuições para a constituição de novas formas de organização do trabalho em saúde. Rev. Bras. Enferm. 2009; 62(6):863-869.

30. Cecílio LCO. Autonomia versus controle dos trabalhadores: a gestão do poder no hospital. Cien Saude Colet 1999; 4(2):315-329.

31. Weber L, Grisci CLI. Trabalho, gestão e subjetividade: dilemas de chefias Intermediárias em contexto hospitalar. Cad. EBAPE.BR. 2010; 8(1):54-70.

32. Cabana MD, Rand CS, Powe NR, Wu AW, Wilson, $\mathrm{MH}$, Abboud PAC, Rubin HR. Why don't physicians follow clinical practice guidelines? JAMA 1999; 282(15):1458-1465.

33. Leentjens AF, Burgers JS. What factors are important for the successful implemation of guidelines? Tijdschr Psychiatr. 2008; 50(6):329-335.

34. Forsner T, Wistedt AA, Brommels M, Forsell Y. An approach to measure compliance to clinical guidelines in psychiatric care. BMC Psychiatry 2008; 8:64.

35. Grol R, Grimshaw J. From best evidence to best practice: effective implementation of change in patient's care. The Lancet 2003; 362(9391):1225-1230.

36. Organização Mundial de Saúde (OMS). Organização Pan-americana de Saúde. El papel del farmacêutico en el sistema del atención de salud. Tóquio: Informe de La Reunión de la OMS; 1993.

Artigo apresentado em 09/05/2011

Aprovado em 03/06/2011

Versão final apresentada em 18/06/2011 